\title{
Tissue Attenuation Characteristics of Acoustic Emission Signals for Wear and Degradation of Total Hip Arthroplasty Implants
}

\author{
Ataif Khan-Edmundson* Geoffrey W. Rodgers**** \\ Tim. B.F. Woodfield $* *$ Gary J. Hooper**, and J. Geoffrey Chase* \\ *Dept. of Mechanical Engineering, University of Canterbury, Christchurch, New Zealand 8140 \\ (Tel: 64-3-3642596; e-mail: geoff.rodgers@ canterbury.ac.nz,geoff.chase@canterbury.ac.nz). \\ **Dept. of Orthopaedic Surgery and Musculo-Skeletal Medicine, University of Otago Christchurch, New Zealand \\ (e-mail: tim.woodfield@otago.ac.nz)
}

\begin{abstract}
Recent research has investigated the use of Acoustic Emission (AE) monitoring of patients with Total Joint Replacement (TJR) implants. This technique involves using a set of four passive ultrasonic receivers to monitor the acoustic events that are created when a TJR implant is articulated through a range of motion. Both in-vitro and in-vivo monitoring of implants is possible. The soft-tissue attenuation characteristics are a very important aspect of how these two signal types are related as the aim of $\mathrm{AE}$ monitoring is to provide in-vivo diagnosis of implant degradation. This manuscript presents the results of in-vivo monitoring of patients with Total Hip Replacement (THR) implants. The corresponding Bode plots are presented to approximate the soft tissue attenuation characteristics. Overall averages are taken across 45 patient data sets and each of the four sensors, located against the skin surface, from the greater trochanter to mid-femur. Each sensor set is also analysed individually to delineate different tissues attenuation at the different locations. These results of this research can be used to determine the maximum likely frequency of interest present on the skin surface during $\mathrm{AE}$ monitoring, even if higher frequencies may be observed in-vitro.
\end{abstract}

Keywords: Biomedical Systems, Vibration Measurement, Vibration Monitoring, Ultrasonic Transducers, Signal Analysis, Bode Plot, Frequency Spectrum, Attenuation Observations, Acoustic Emissions, Orthopaedic Implants, Patient Testing, Tissue Attenuation.

\section{INTRODUCTION}

The number of total knee replacements performed in the U.S. will leap by $673 \%$ - reaching 3.48 million - by the year 2030 , and hip replacements will increase by $174 \%$ to 572,000 (Kurtz et al., 2003), largely due to demographic ageing (NZOA, 2003). Total joint replacement (TJR) surgery is typically the last resort for people with osteoarthritis (OA), also known as degenerative joint disease. TJR surgery is extremely successful ( $90 \%)$, but these joints need to be replaced due to wear and/or premature loosening of the implant after 10-15 years (Kurtz et al., 2003; NZOA, 2003). The more primary joint replacements surgeries there are, the more revision TJR surgeries there will be all else equal, thus creating a significant and increasing cost, in both dollars and use, of scarce surgical services.

With an epidemic of degenerative joint disease occurring, there is a huge challenge to find and implement effective screening programmes for detecting early TJR wear or failure, and clear diagnostic indicators for orthopaedic surgeons to properly manage revision surgery (NZOA, 2003; Browne et al., 2005). Early diagnosis of impending failure can save significant time, cost and more serious surgery. Currently, there are no reliable, non-traumatic and noninvasive methods to monitor the healing process or loosening status after TJR.
Research over the past 15-20 years has investigated acoustic emission (AE) monitoring to provide insight into implant condition and provide early detection of wear and loosening (Browne et al., 2005). AE monitoring devices use passive ultrasonic receivers to record high frequency vibrations emitted by the implant and correlate the recorded signal with clinical outcomes. The ultrasonic signals are typically characterised on frequency content or signal characteristics (short-duration high amplitude events/long-duration, lower amplitude events). These AE signals can be correlated with events, such as micro-scale brittle breakages of bone or bone cement, or vibrations due to wear and/or wear debris within the bearing surface between the femoral and acetabular components. Previous in-vitro and in-vivo studies demonstrate the potential $\mathrm{AE}$ frequency range of interest varies significantly (up to $1 \mathrm{MHz}$ in-vitro, but only up to $50 \mathrm{kHz}$ for in-vivo tests on the skin surface) due to attenuation of vibrations through tissue (Browne et al., 2005) and there has been no research explicitly looking at the tissue attenuation characteristics. Moreover, AE monitoring devices typically utilise a single sensor located near the greater trochanter to determine joint condition.

Recent research has developed an AE prototype diagnostic tool to assess implant designs and materials. The prototype includes four ultrasonic sensors placed against the skin, between the greater trochanter and the mid femur. The additional information from the multiple sensors and relative 
signal strength at each location can determine likely vibration sources (acetabular cup, bearing surface or femoral stem) and lead to clinical diagnosis. The technology can be applied to cemented versus uncemented components, and the performance of metal-on-plastic, metal-on-metal, and ceramic-on-ceramic bearing surfaces, and combinations thereof. This manuscript aims to investigate the range of frequencies observed on the skin surface during patient testing and to develop a soft tissue attenuation model.

\section{METHODS}

An AE prototype was used to undertake in-vivo monitoring of patients with Total Hip Replacement (THR) implants. The prototype consisted of four passive ultrasonic receivers, each with a resonant frequency of $25 \mathrm{kHz}$. The ultrasonic sensors were placed against the skin, between the greater trochanter and the mid femur The data from each sensor was simultaneously recorded at $100 \mathrm{kHz}$ as the patients undertook a range of standard orthopaedic test motions. These included standing from sitting in a chair, dropping from standing to crouching, and walking up stairs. The recording system was set to trip and record a data frame in response to any sensor exceeding a nominal voltage threshold. A total of 45 patients were tested, with some patients having unilateral implants and others having bilateral implants. Those with bilateral implants had both hip implants monitored independently.

This research evaluates this prototype data. The main goal is to assess concept feasibility. In particular, it seeks to determine the transfer function and attenuation properties between the impulse sources and the measured response at the sensors. These first analyses will show the feasibility of the overall concept.

The analysis methodology is focused on first investigating the time domain response and determining whether intrapatient repeatability is possible across the range of patient input actions (standing from sitting etc.). The signals are then evaluated individually in the frequency domain by the use of Fourier transforms. Finally, Bode plots are generated in an overall average sense and on a per-sensor basis, to determine tissue attenuation characteristics. In these Bode plots the output signal frequency spectrum is used to approximate the transfer function based on the assumption that the signal generation at the source can be modelled as an impulse input with broad input frequency content.

\section{RESULTS}

\subsection{Time Domain Results of Patient Trials}

Fig. 1 presents a typical time-domain response of a patient trial. In some cases the source of the vibration can be indicated by a phase delay between when the signal is detected at each of the four sensors. In Fig. 1 it is evident that the likely source of the vibration is at the lower end of the implant and may be due to loosening of the femoral component. This observation is due to the vibration being first detected on the lower sensor, followed by the upper sensors.

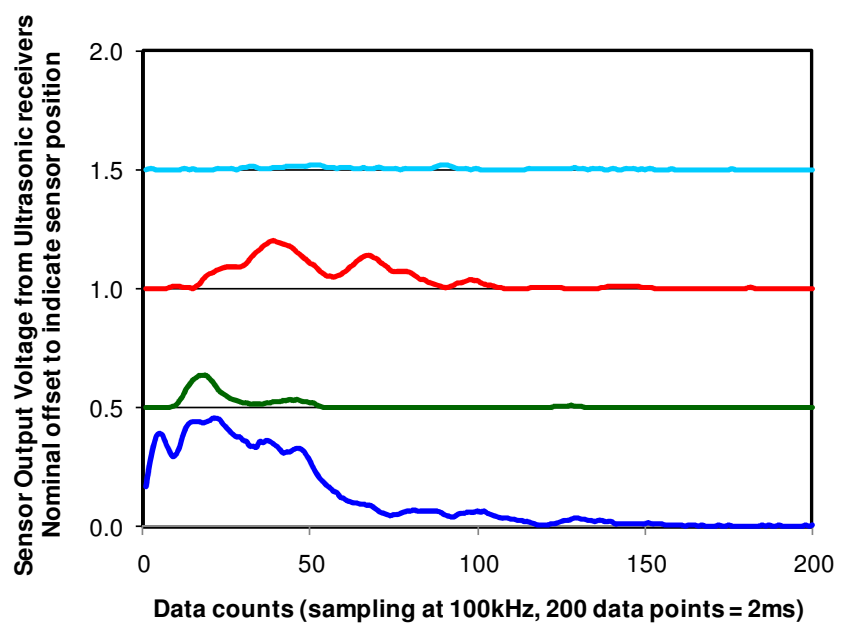

Fig. 1. Typical time-domain response of an in-vivo patient trial. Vertical scale is volts, with a nominal voltage offset for each sensor to indicate position.

Intra-patient repeatability is a key aspect of the response and a very important consideration if discrimination between failure modes is to be achieved. Fig. 2 presents a group of repeated tests for a patient undertaking a range of different tasks. It is evident that intra-patient repeatability is present for this patient and that even a range of different tasks leads to the same distinct dominance of one sensor. Note that Figure 2 represents a different patient to those in Figure 1.

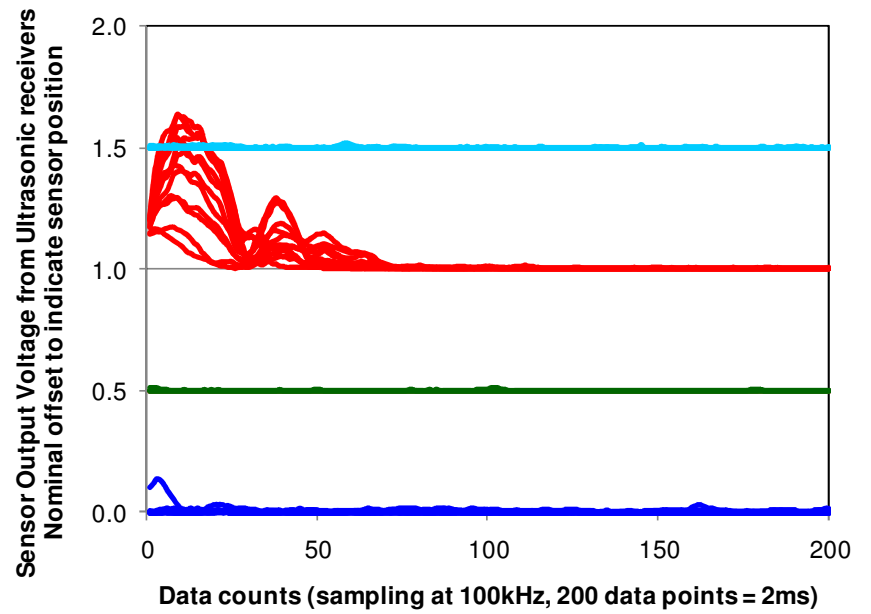

Fig. 2. Repeated tests for a single patient to test intra-patient variability. As in Fig. 1, the vertical scale is volts, with a nominal voltage offset for each sensor to indicate position.

\subsection{Frequency Domain Results}

The time domain results for each patient were obtained through a discrete fourier transform. It was initially expected that most of the content in the signals would be well above the range of human hearing and generally at least $20 \mathrm{kHz}$. However, the patient trial results showed that the signals were almost exclusively below $15 \mathrm{kHz}$, with a vast majority 
of the signal below $5 \mathrm{kHz}$. Fig. 3 presents a typical frequency domain magnitude plot of the measured in-vivo patient data.

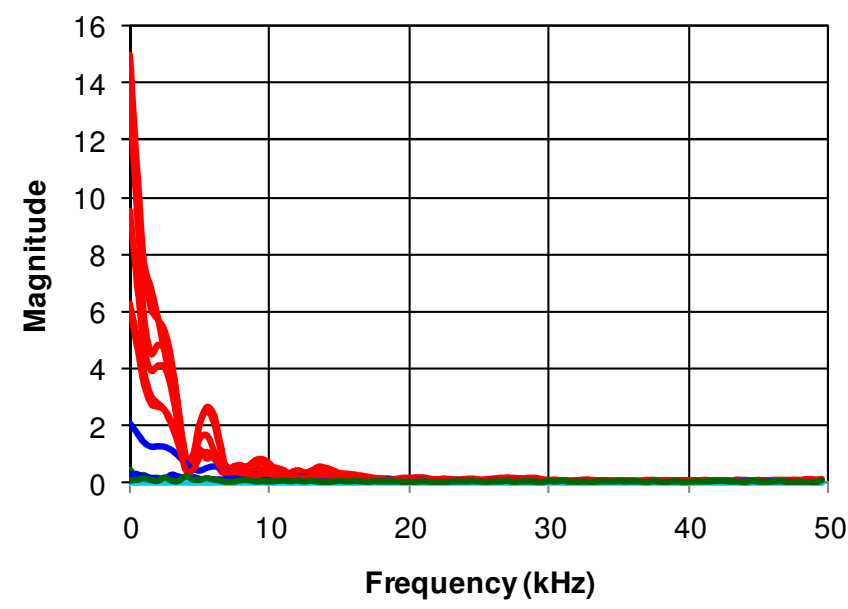

Fig. 3. Frequency domain magnitude of in-vivo signals. Note that the data and series colours correspond to those presented in Fig. 2.

It is expected that the actual in-vitro acoustic emissions at the implant will produce much higher frequencies than those observed in Fig. 3. When considering an event such as the breakage of bone or bone cement, or impacting of the implant bearing surfaces, it is expected that the signal generation could be considered as an impulse input. Therefore, the range of frequency content produced at the implant would be expected to cover a much wider frequency band than seen at the skin surface. Therefore, it was considered that the frequency range at the skin surface, if averaged over a range of patients, would provide a good estimate of the overall tissue attenuation/transmission properties.

Hence, the frequency spectrum was plotted on a log-log scale as a Bode plot, typical for investigating a transfer function. Fig. 4 presents the Bode plot for a total of 45 patients. Fig. 4 shows one line per patient for each of the 45 of the patients in the clinical trial. To produce this plot, the data in each channel was averaged (all 200 data point frames were averaged together), and the channels were also averaged together. This approach gave one line per patient, as shown in Fig. 4. Note that it is assumed that the input is an impulse. Therefore, the output spectrum can be directly considered as the transfer function.

It is evident in Fig. 4 that there is a consistent fall-off in magnitude beyond $1 \mathrm{kHz}$. Due to the consistent nature of this drop in magnitude and the assumption of an impulse input, it is assumed that this fall-away represents the soft-tissue attenuation. However, also evident in Fig. 4 is the variable signal amplitude at low frequencies with values ranging from -30 to $-55 \mathrm{~dB}$. This observation can be explained easily by considering that all of the signal magnitudes are variable and that the overall magnitude of the acoustic events at the implant varies accordingly. Equally, it may represent interpatient variability.

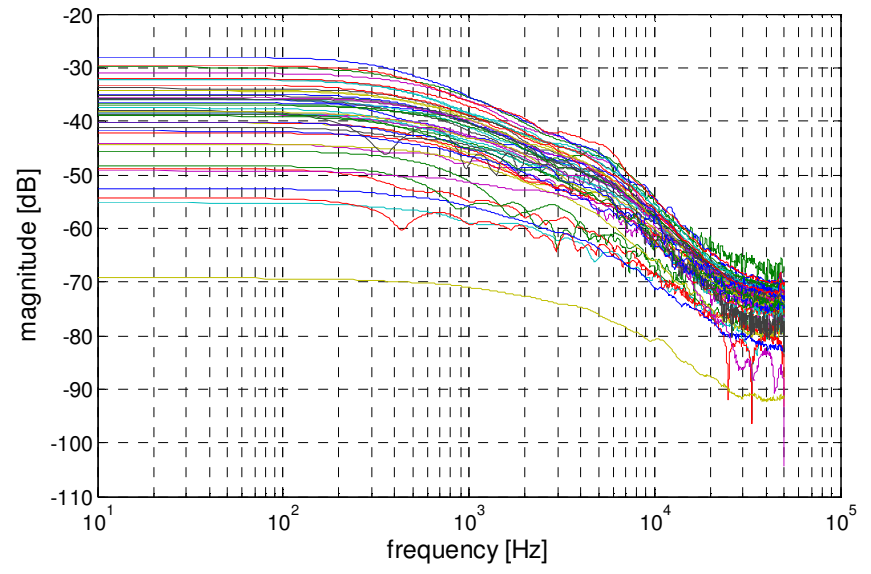

Fig. 4. Bode plot of the in-vivo patient data.

To eliminate this affect, all of the responses on the Bode plot of Fig. 4 were normalised to have the same initial magnitude. This normalisation enables easier comparison of the relative attenuation at higher frequencies. The results are presented in Fig. 5.

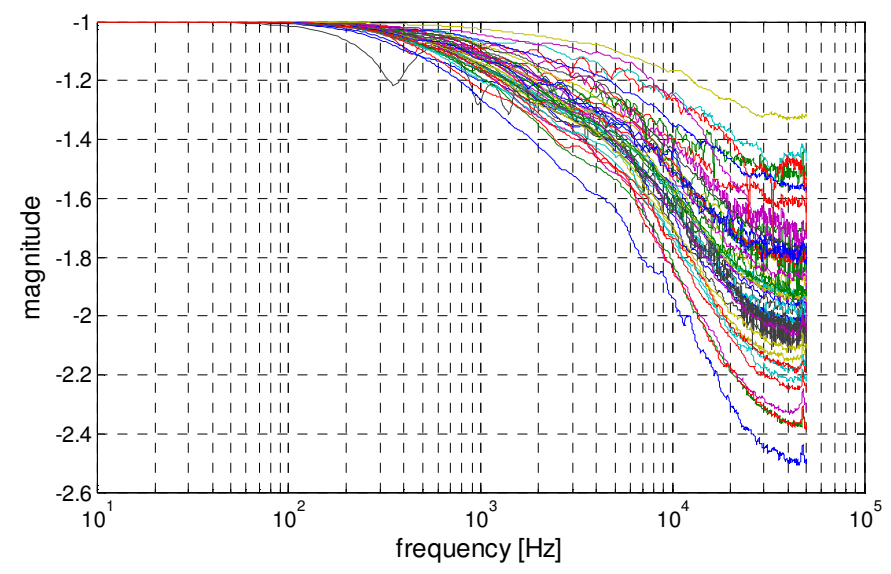

Fig. 5. Bode plot of the in-vivo patient data, with responses normalised to the same initial magnitude.

It can be seen in Fig. 5 that the exact attenuation characteristics appear more variable, but that the relative frequency content drops off significantly at the higher frequencies, irrespective of peak signal magnitude.

It should also be noted that the responses in Figs. 4 and 5 are averaged across all patient trials and all sensor locations. It is expected that the signal attenuation characteristics will very across each sensor as there is different underlying tissue at each sensor location. It might be expected that the sensor placed near the greater trochanter will exhibit less attenuation as the skin surface is near a bony landmark and there is less soft tissue for the signal to transmit through. Conversely, the sensors on the mid-femur have much more muscle, fascia, and soft tissue to attenuate the response. However, given that the input magnitude is not known and there is no way of normalising to get the true transmission, there are some limitations to this analysis and care should be taken when considering the results. 


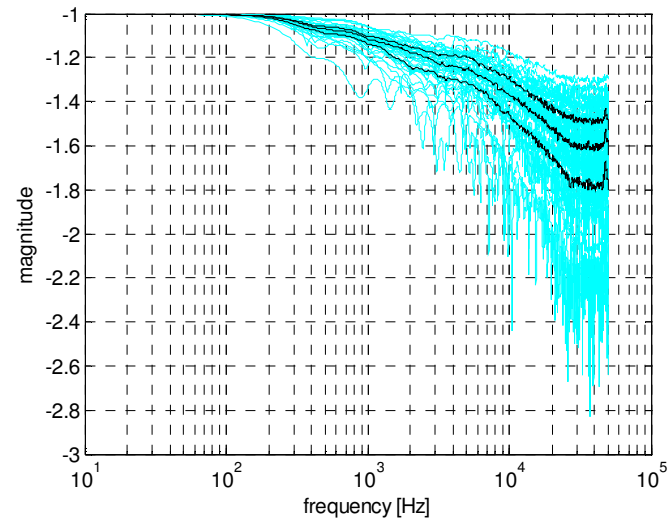

a) Response of sensor 1 (top)

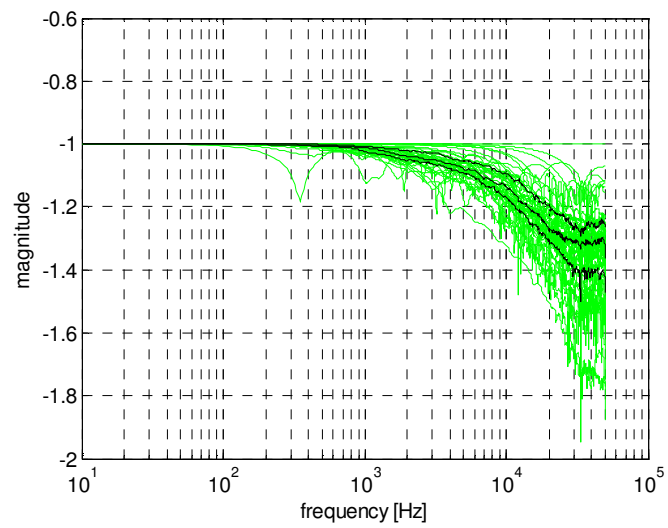

b) Response of sensor 2

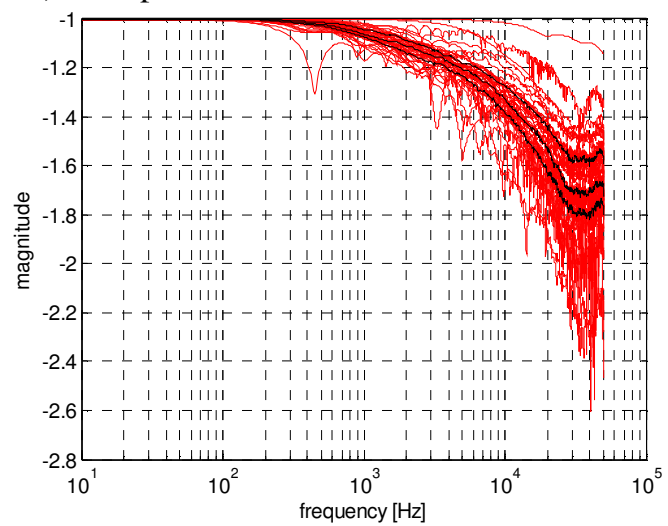

c) Response of sensor 3

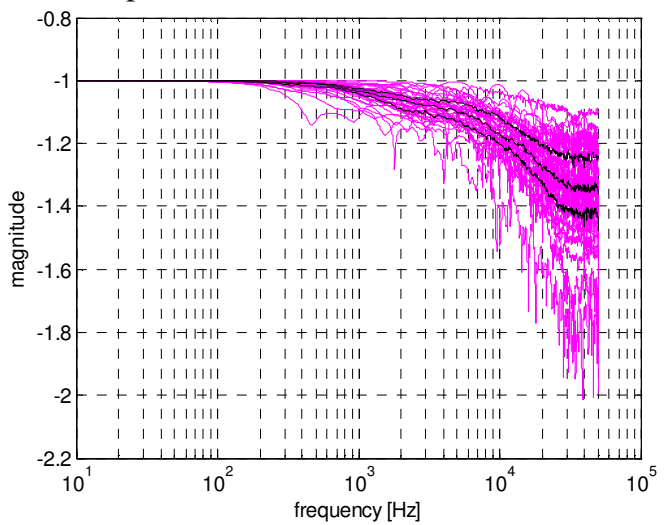

d) Response of sensor 4 (bottom)

Fig. 6. Bode plots of the in-vivo patient data, split into individual sensors, with responses normalised to the same initial magnitude.
Figs. 6a-d present the attenuation characteristics for each sensor independently. The response of each sensor is plotted for each patient and normalised to the same overall magnitude using the same approach as that of Fig. 5. Fig. 6 is essentially Fig. 5, but split into the responses from each of the four sensors. Also shown in Fig. 6 are the median, 25th and 75 th percentile lines to give an indication of the spread of the data. It is evident in Fig. 6 that the attenuation characteristics are broadly similar for all of the sensor locations. Specific differences can be attributed to differences in the underlying soft tissue make-up at the sensor locations or, more specifically, inter-patient variability.

To better compare the attenuation characteristics of each sensor location, all of the median, $25^{\text {th }}$ and $75^{\text {th }}$ percentile lines from Figs. 6a-d have been reproduced on the same axes in Fig. 7. It can be seen in Fig. 7 that the sensors do show some clear differences in their specific attenuation characteristics. Sensors 1 and 3 show similar attenuation characteristics above $20 \mathrm{kHz}$, with both of these sensors showing much lower transmission than sensors 2 and 4 . Sensor 1 shows the lowest transmission of any of the sensors between $100 \mathrm{~Hz}$ and $10 \mathrm{kHz}$. This is not an expected result, as this sensor is up near the greater trochanter and it is assumed that the greatest transmission would occur at this point. However, there may be other influencing factors, such as less inherent noise generation at the upper acetabular components, compared with the lower femoral components.

Overall, all sensors show a notable drop-off in frequency content above $10 \mathrm{kHz}$. It should be noted that this observation of lower frequency magnitude in this range may be a combination of tissue attenuation and the specific characteristics of the ultrasonic receivers. However, the ultrasonic receivers had a design resonant frequency of $25 \mathrm{kHz}$, so it is expected that any frequency content present at the skin surface in this range $(10-25 \mathrm{kHz})$ would be well detected by the sensors. Therefore, it is reasonable to conclude that even though there may be much higher frequencies created during articulation of the implant interfaces at the source, that very little of these higher frequency vibrations are transmitted to the skin surface.

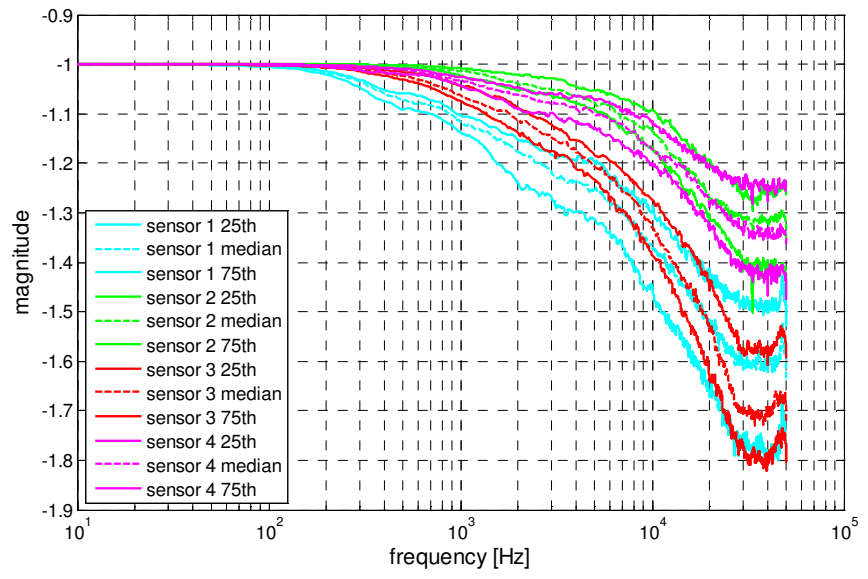

Fig. 7. Combined Bode plot of the in-vivo patient data, split into individual sensors, with responses normalised to the 
same initial magnitude. Each sensor is represented by the $25^{\text {th }}, 50^{\text {th }}$ (median) and $75^{\text {th }}$ percentile.

\subsection{Transfer Function Approximation}

To extend the analyses, it is useful to develop an approximate transfer function of the soft tissue. This transfer function will enable approximation of the likely in-vivo frequencies at the skin surface that can be expected for any observed frequency range during in-vitro testing of the implant. To investigate the actual transfer function, the results of Fig. 7 are presented again in Fig. 8, but without the normalisation to the same initial magnitude. It is evident in Fig. 8 that there is a notable spread as inter-patient variability on the data and that any fitted transfer function must be used with caution. However, the overall results of significant attenuation over $10 \mathrm{kHz}$ remains valid.

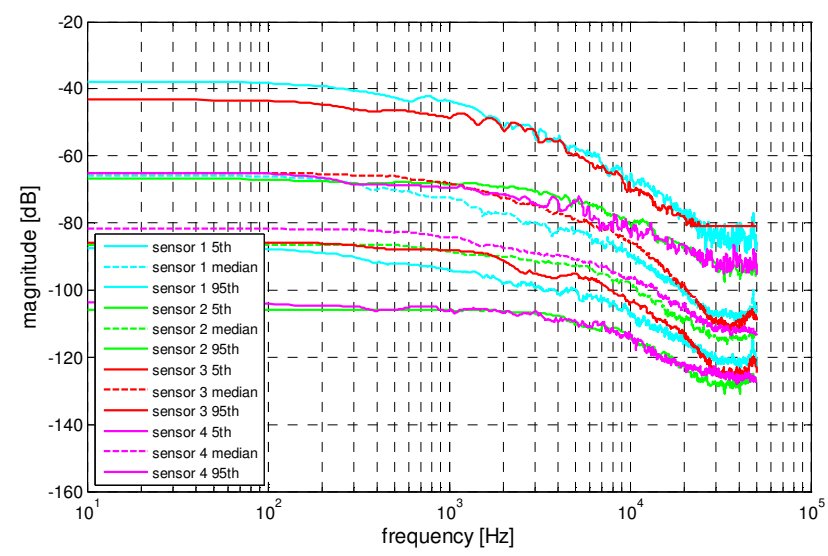

Fig. 8. Combined Bode plot of the in-vivo patient data, split into individual sensors. Each sensor is represented by the $25^{\text {th }}, 50^{\text {th }}$ (median) and $75^{\text {th }}$ percentile.

Fig. 9 presents the transfer function fitted to the patient results. It should be noted that while there is a phase plot in Fig. 9, no phase plots are presented for the patient data. The input for the patient data is assumed to be essentially an impulse input and therefore there is no inherent definition of phase as applied to the patient data, where analytical Bode plots assume sine wave inputs. If the attenuation characteristics were determined from sinusoidal input waveforms, then phase plots could be included. However, given the nature of the system, when the phase response is plotted for the patient data, it appears as a very noisy data set, based around a central value of zero.

Equation 1 describes the empirically fitted transfer function, which is plotted in Fig. 9. Equation 1 is determined based upon a best-fit to the overall patient data and can be used as a rough approximation to the tissue attenuation characteristics. $\mathrm{K}_{\mathrm{o}}$ is a scaling constant, while $\mathrm{z}_{\mathrm{i}}$ are the zeroes of the transfer function and $\mathrm{p}_{\mathrm{i}}$ are the pole locations.

$\mathrm{K}_{0} \frac{\left(\mathrm{s}+\mathrm{z}_{1}\right)\left(\mathrm{s}+\mathrm{z}_{2}\right)}{\left(\mathrm{s}+\mathrm{p}_{1}\right)\left(\mathrm{s}+\mathrm{p}_{2}\right)}=0.0002 \frac{(\mathrm{s}+13000)^{2}}{(\mathrm{~s}+700)(\mathrm{s}+1700)}$

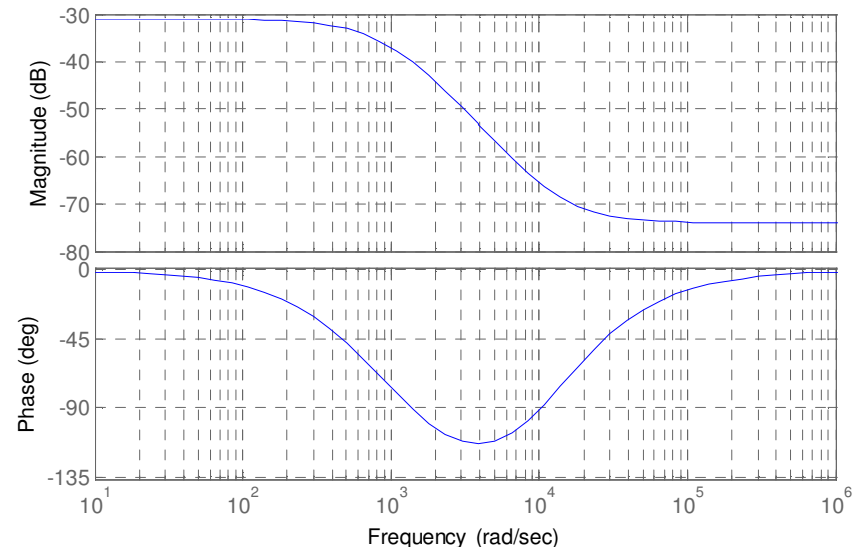

Fig. 9. Analytical transfer function fitted to the patient results.

\section{DISCUSSION}

The development of an Acoustic Emission monitoring device has the potential to be a very useful diagnostic tool for orthopaedic surgeons. The underlying premise of the AE monitoring device is that different wear and failure modes of the implant will produce unique frequency signatures, which can be identified during in-vitro testing of the implants. The AE monitoring device can then be utilised for in-vivo patient testing, when the passive ultrasonic receivers can be placed against the skin surface. When considering the correlation between the frequency response in-vitro and in-vivo, the tissue attenuation properties must be considered. These results of Figs. 3-8 show that while there may be high frequency vibrations present within the response of the implant, these high frequencies are heavily attenuated by the soft tissue. Therefore, the maximum frequency of interest at the skin surface is approximately $20 \mathrm{kHz}$, and in practice most of the content is well below $10 \mathrm{kHz}$.

It should be noted that much of the data presented here dates back to orthopaedic implants, utilising primarily the metalon-plastic and metal-on-metal bearing surfaces. Newer, ceramic-on-ceramic implants have the potential to produce higher frequency vibrations as well as audible squeaking of the implants. Ongoing research is undertaking a new range of patient trials, testing patients with a range of implant bearing surfaces. It should also be noted that the source of vibration from the implants is not restricted to just the bearing interface. Any loosening of the femoral head on the morse taper, looseing of the femoral stem within the femur, or loosening of the acetabular components will all produce a vibration response. The ongoing research attempts to correlate the vibration measurements with diagnostic indicators via two key, parallel methods. In-vitro testing is being undertaken to identify any unique frequency responses that can be attributed to movement of different implant interfaces. These identified frequencies can then be considered with respect to the attenuation model presented here, to estimate the in-vivo frequencies of interest. 
It should be noted that these results are specific to hip replacement implants, but that a similar approach could be used for knee replacements. However, the attenuation properties will likely be quite different due to the proximity of the boney landmarks to the skin surface.

\section{CONCLUSIONS}

This manuscript presents the in-vivo patient testing results of an AE monitoring device prototype. Assuming that the vibration range produced within the implant can be approximated as an impulse, with broad frequency content, the output frequency spectrum has been used to approximate the soft tissue attenuation characteristics. The results indicate that the maximum frequencies present at the skin surface are approximately $20 \mathrm{kHz}$, with a vast majority of the signal being well below $10 \mathrm{kHz}$. Ongoing research continues to investigate and further verify the frequencies of interest in the response of orthopaedic implants.

\section{REFERENCES}

Browne M, Roques A, Taylor A. The acoustic emission technique in orthopaedics - a review. Journal of Strain Analysis for Engineering Design 2005; 40(1): 59-79

Kurtz S, Ong K, Lau E, Mowat F, Halpern M. Projections of primary and revision hip and knee arthroplasty in the United States from 2005 to 2030. J Bone Joint Surg Am 2007; 89(4): 780-785.

New Zealand Orthopaedic Association (NZOA). The Ageing of New Zealand - An Epidemic with Major Impact On Musculo-skeletal Disease, 2003.

Rothwell AG, Hobbs T, Hooper GJ, New Zealand Orthopaedic Association (NZOA). National Joint Register Nine Year Report (www.cdhb.govt.nz/njr/), 2008. 